\title{
Pain recognition and scales for livestock patients
}

\begin{abstract}
No longer is it acceptable to say that farm animals do not need analgesic care. An understanding of pain, physiology, signs of pain unique to livestock and conditions that may require analgesic care are necessary for any veterinary nurse working with livestock species. It is the veterinary nurse's duty to recognize and assess pain in livestock species to enable better clinical care for their patients. Education for the owner/farmer and entire veterinary staff about pain in livestock is critical.
\end{abstract}

\author{
Volume 7 Issue 5 - 2018

\begin{abstract}
Mary Ellen Goldberg
Veterinary Pain PractitionerVTS - lab animal medicine (research anesthesia) VTS-physical rehabilitation, International veterinary academy of pain management, USA
\end{abstract}

\begin{abstract}
Correspondence: Mary Ellen Goldberg, Veterinary Pain PractitionerVTS - lab animal medicine (research anesthesia) VTS-physical rehabilitation, International veterinary academy of pain management, USA, Email mewhitester@gmail.com
\end{abstract}

Received: June 25, 2017 | Published: November 19, 2018

\section{Introduction}

Livestock patients for this lecture will include cattle, sheep, goats and pigs. Animal welfare has increased the importance of pain management in livestock. Multimodal analgesia is the preferred method of pain relief for even minor surgical procedures. Animal suffering is no longer tolerated nor is the unwillingness by owners to pay for medications. ${ }^{1}$ Dr. Temple Grandin's work in the livestock industry on animal behavior has shown that animals (livestock) are not just things to be owned, but instead they can feel pain and suffer. ${ }^{1}$ Veterinary technicians and nurses have the ethical duty to advocate for these patients to the same standard as any others.

\section{Recognition of pain in cattle}

Cattle in pain often appear dull and depressed, with the head held low and showing little interest in their surroundings. There are in appetence, weight loss, and, in milking cows, a sudden drop in milk yield. On being handled, cows may react violently or adopt a rigid posture designed to immobilize the painful region. Grunting and grinding of the teeth may be heard. Acute pain may be associated with bellowing. Generally, signs of abdominal pain are like those seen in the horse but are less marked. Rigid posture may lead to a lack of grooming due to unwillingness to turn the neck. In acute abdominal conditions, such as intestinal strangulation, the animal adopts a characteristic stance with one hindfoot placed directly in front of the other. At a certain level of severity, pain in ruminants can be identified easily because an animal is unable to hide the experience of pain or the physical insult causing the pain may interfere with normal function (e.g., lameness) (Figure 1). ${ }^{2}$

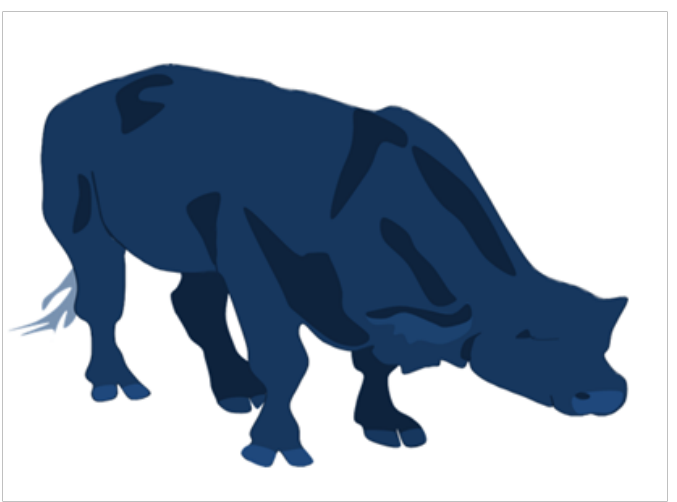

Figure I Cattle pain stance

\section{Recognition of pain in sheep and goats}

Sheep often only show subtle signs of pain, while goats are intolerant of painful procedures. ${ }^{1}$ Goats will often bleat, while sheep may only exhibit tachypnea, inappetence, grinding of teeth, immobility, or abnormal gait. Following procedures such as castration and tail docking, lambs may show signs of discomfort such as standing up and lying down repeatedly, tail wagging, occasional bleating, neck extension, dorsal lip curling (Flehman), kicking, rolling and hyperventilation. Sheep are a timid prey species and unlikely to advertise that they are compromised; thus, one would expect overt signs of pain only after seriously painful procedures or experiences. Moreover, unlike cattle and goats, sheep tend to remain silent rather than vocalizing during painful procedures. ${ }^{2}$ Goats are more vocal animals than sheep or cattle. Kids commonly hide and wait for their mother to return and feed them, unlike lambs, which follow their mother all the time. This may explain the difference in the behavior of kids and lambs after castration.

\section{Recognition of pain in pigs}

Pigs in pain may show changes in gait and posture. They normally squeal and attempt to escape when handled; however, these reactions may be accentuated when the animal is in pain. Adult pigs may become aggressive. Squealing is also characteristic when painful areas are palpated. Handling of chronic lesions may not elicit signs

of pain. Pigs will often be unwilling to move and may hide in bedding if possible. ${ }^{1}$ Pigs are stoic animals, but are also a prey species inclined to mask signs of illness, weakness, pain, or vulnerability until they can no longer compensate. Decreased food intake, as well as less time spent eating, has been well documented in painful or ill swine of all ages. Not all ill or painful pigs stop eating, but all pigs that stop eating are likely to be suffering. ${ }^{2}$ Decreased activity is an important sign of pain or discomfort in an animal like the pig that normally spends a large part of its day foraging and exploring can be an important sign of pain or distress. Pigs with forelimb arthritis frequently stand in a hunched position with more weight on the pelvic limbs. Lying and rising may be more painful than simply standing in a position in which pressure on the sore limb or limbs can be relieved. Pigs in pain may also be stiff, tremble, or isolate themselves from other pigs and people. Pigs with visceral pain may kick at the abdomen or may shift repeatedly when lying down, suggesting that they cannot get comfortable. Potential causes of chronic pain, such as neoplasia or severe infectious or degenerative processes, are often discovered late during the disease and typically lead to euthanasia. Arthritis is a 
primary example of a chronic pain condition in the pet pig.

\section{Pain scales for livestock}

\section{Cattle}

In surveys of veterinarians concerning the use of analgesics in cattle practice, lack of knowledge in recognizing pain, ${ }^{3}$ the belief that farm animals feel less pain than smaller animals, ${ }^{4}$ economic reasons ${ }^{5,6}$ and the lack of valid

and reliable instruments to assess pain have been cited as the main reasons why analgesics are not used more frequently. ${ }^{3}$ In cattle, a scoring system for gait has been validated and found to be reliable and sensitive for identifying cows with severe hoof lesions. ${ }^{7}$ The UNESPBotucatu unidimensional pain scale for assessing acute postoperative pain in cattle is a valid, reliable and responsive instrument with excellent internal consistency and

discriminatory ability. The cut-off point for rescue analgesia provides an additional tool for guiding analgesic therapy. ${ }^{8}$ The Cow Pain Scale included, 'attention towards the surroundings', 'head position', 'ears position', 'facial expressions', 'response to approach' and 'back position'. Further-more, treatment with a systemic analgesic significantly reduced the pain score of the group, where clinical examination suggested pain but did not affect the cows in the control group. Taken together, these results suggest that the Cow Pain Scale (CPS) may be used to identify cows in pain (Figure 2). ${ }^{9}$

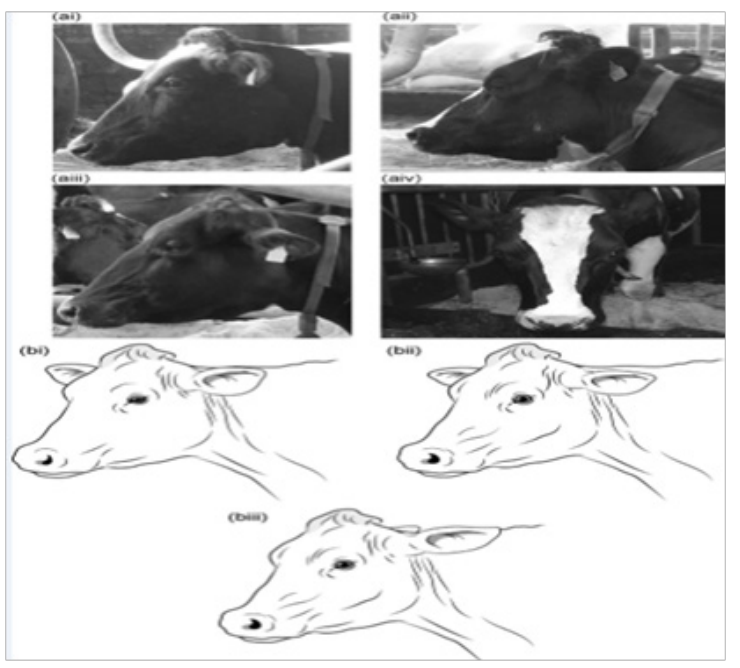

Figure 2 Dairy cow grimace scale

\section{Sheep}

Sheep have been studied to assess their pain. A study revealed that sheep that underwent maxillofacial surgery displayed postoperative hypersensitivity lasting at least three days after surgery, despite intra and postoperative treatment with opioids, an NSAID and local anesthesia. ${ }^{10}$ Another scale looked at locomotion in sheep. ${ }^{11}$ The results indicate that the locomotion scoring scale using groups of defined observations for each point on the scale was reliable and may be a useful research tool to identify and monitor locomotion in individual sheep when used by trained observers. The use of facial expression scoring to assess pain is a well-utilized, practical tool in both humans and non-human animals. Facial expression as a pain scoring method offers the potential to start to under-stand this side of animal pain. The Sheep Pain Facial Expression Scale (SPFES) provides an accurate and reliable method to recognize and assess pain in sheep (Figure 3). It also doubles as a training tool for veterinarians and farmers to learn more about changes in the facial expression of sheep when they are likely to be suffering from pain. Such a tool is likely to improve an observer's ability to quantify pain in animals and allow observers to discriminate between different pain states independent of disease status, as well as detect the effectiveness of pain relief. Prompt recognition of pain using the scale will enable farmers and veterinarians to treat and manage their flocks better, reducing the impact of pain on their sheep, thus improving welfare and production. ${ }^{12}$



Figure 3 Sheep grimace scale

\section{Goats}

Stockpeoples' ability to recognize pain in their livestock, and to respond appropriately, is of utmost importance for animal welfare. Assessment of pain is complex, and attitudes and empathy are thought to play a role in peoples' responses to the sight of pain. The Norwegian dairy goat has been utilized to assess the ability of stockpeople to evaluate pain. ${ }^{13}$ A Numeric Rating Scale (NRS) for assessment of post-operative pain for Goats has been developed. ${ }^{14}$ 


\section{Pigs}

There are a couple of studies that have undertaken to assess a Grimace Scale for Pigs. ${ }^{15}$ The Piglet Grimace Scale requires considerable further development as a potential tool to detect postprocedural pain in neonatal pigs. ${ }^{16}$ Full characterization of the Piglet Grimace Scale would give the opportunity to implement it as a costeffective tool for the on-farm assessment of painful and or distressing conditions induced by husbandry in piglets. Finally, it would provide the basis for the development of a scale specific to non-neonatal pigs, which would benefit the assessment of pain in relation to spontaneous health conditions (e.g., lameness induced by degenerative joint diseases or infection) that are normally observed in older animals. A dissertation was done in Uppsala, Sweden. ${ }^{17}$ The findings in this study suggest that also in pigs, a pain face can be identified. This porcine pain face seems to comprise an angled appearance of the area above the eyes, wrinkling of the snout, and tension of muscles around the mouth and along the lateral side of the head, as well as lowered ears held in an asymmetrical manner or completely backwards (Figure 4). These features were sometimes rather subtle however, and the method may thus be difficult to apply in a clinical situation. Further studies are required to evaluate and develop the porcine pain face, which may then possibly constitute a valuable additional pain assessment tool in pigs.

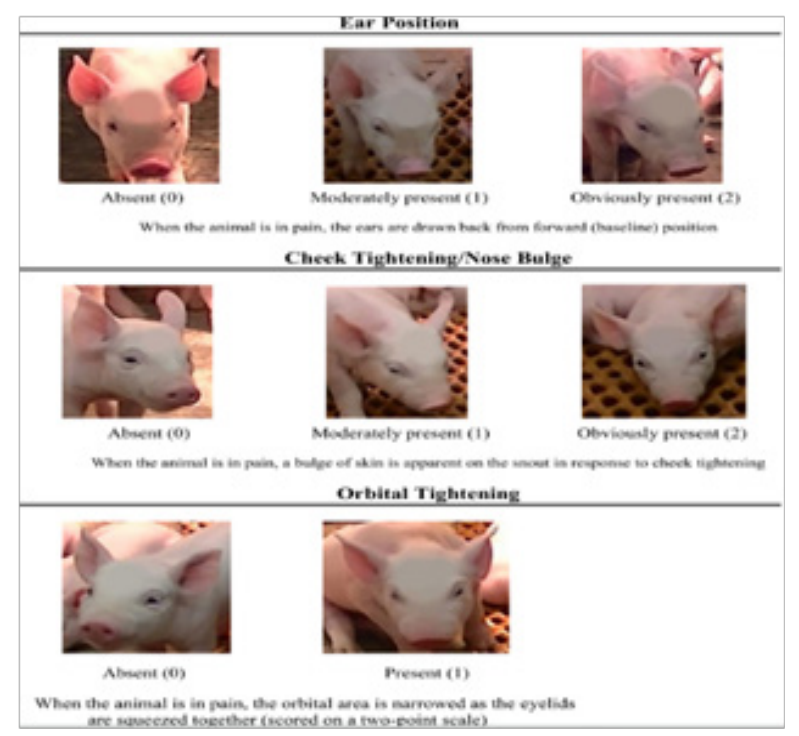

Figure 4 Piglet grimace scale

\section{Procedures that cause Pain in cattle ${ }^{\prime}$}

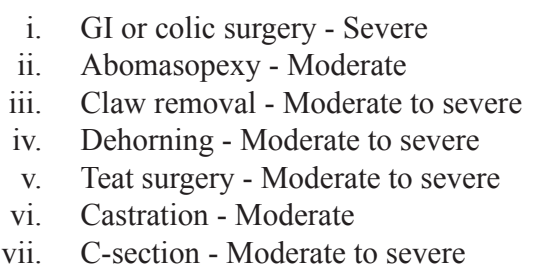

\section{Procedures that cause pain in sheep and goats'}

i. C-section - Moderate to severe

ii. Perineal Urethrostomy - Moderate to severe

iii. Castration - Moderate

iv. Dehorning - Moderate to severe

v. Claw removal - Severe

\section{Typical procedures that require analgesia in pigs'}

i. Castration: baby pigs, pet pigs, and adult or larger pigs

ii. Aural hematoma

iii. Caesarean section

iv. Amputation: digital

v. Amputation: uterus

vi. Atresia ani

vii. Entropion: frequent in pot-bellied pigs

viii. Exploratory laparotomy

ix. Fractures

x. Hernia: inguinal and umbilical

xi. Hoof trimming

xii. Joint lavage

xiii. Mastectomy

xiv. Ovariohysterectomy

xv. Prolapse: rectal, vaginal, uterine

xvi. Tail docking

xvii. Tooth clipping

xviii. Tusk removal

xix. Cryptorchid

xx. Vasectomy

\section{Medications used in livestock}

When considering pain management strategy, one should contemplate prevention (preemptive) and control of those pain pathways already activated in nociception. ${ }^{18}$ Most commonly used medications in Cattle are NSAIDS, opioids, local techniques such as epidural anesthesia, intravenous regional anesthesia (IVRA), and the use of local nerve blocks provide analgesia to specific areas, Alpha2-Adrenergic agonists, Ketamine, and Analgesic Adjuvants. Constant Rate Infusions (CRIs) have been described. ${ }^{1}$ Most commonly used medications in Sheep and Goats are nsaids, Opioids, Alpha-2Adrenergic Agonists, nmda Receptor antagonists (Ketamine), Local Anesthetic blocks and infusions and Analgesic Adjuvants. CRIs have been utilized in small ruminants. ${ }^{1}$ Most commonly used medications in Pigs are Opioids, nsaids, Alpha-2-Adrenergic Agonists, nmda Receptor antagonists (Ketamine), Local Anesthetic blocks and infusions and Analgesic Adjuvants. CRIs have been commonly utilized in pigs. ${ }^{1}$

\section{Withdrawal period'}

The withdrawal period is defined as the interval required after dosing for tissue concentrations of a drug or its metabolite to deplete to less than a specific concentration that has been established to be safe for human consumption. The withdrawal period MUST be emphasized in any animal that will enter the food chain.

\section{Conclusion}

Pain Management for Livestock patients is doable. The veterinary nurse must learn about recognition and assessment of pain in these patients. They need to learn how to score pain in cattle, sheep, goats and pigs. They can teach veterinary staff and owners about this pain. They can even help the owners learn how to score pain in their livestock. If the owner does not have a pain scale that they like, then the veterinary nurse can develop a pain scale that the owner/farmer can use. It is best to keep it simple and straight forward.

\section{Acknowledgements}

None 


\section{Conflict of interest}

The author declares that there is no conflict of interest.

\section{References}

1. Kata C, Rowland S, Goldberg ME. Chapter 3 Pain Recognition in Companion Species, Horses and Livestock. Pain Management for Veterinary Technicians and Nurses. Edited by Mary Ellen Goldberg. 2017.

2. Stafford KJ. Chapter 31 Recognition and Assessment of Pain in Ruminants. Pain Management in Veterinary Practice. Edited by Christine M Egger, Lydia Love and Tom Doherty. 2014.

3. Lorena SE, Luna SP, Lascelles BD, et al. Attitude of Brazilian veterinarians in the recognition and treatment of pain in horses and cattle. Vet Anaest Analg. 2013;40(4):410-418.

4. Raekallio M, Heinonen $\mathrm{K}$, Kuussaari J, et al. Pain alleviation in animals: attitudes and practices of Finnish veterinarians. Vet $J$ 2003;165(2):131135 .

5. Anil L, Anil SS, Deen J. Pain detection and amelioration in animals on the farm: issues and options. J Appl Anim Welfare Sci 2005;8(4):261-278.

6. Hewson CJ, Dohoo IR, Lemke KA, et al. Canadian veterinarians' use of analgesics in cattle, pigs, and horses in 2004 and 2005. Can Vet J. 2007;48(2):155-164.

7. Flower F, Weary D. Effect of hoof pathologies on subjective assessments of dairy cow gait. J Dairy Sci. 2006;89(1):139-146.

8. Oliveira FA, Loureiro Luna SP, Barros do Amaral J, et al. Validation of the UNESP-Botucatu Unidimensional composite pain scale for assessing postoperative pain in cattle. BMC Veterinary Research. 2014.

9. Gleerupa KB, Andersenb PH, Munksgaardc L, et al. Pain evaluation in dairy cattle. Applied Animal Behaviour Science. 2015; 171:25-32.
10. Abu-Serriah M, Nolan AM, Dolan S. Pain assessment following experimental maxillofacial surgical procedure in sheep. Laboratory Animals. 2007;41(3):345-352.

11. Kaler J, Wassink GJ, Green LE. The inter- and intra-observer reliability of a locomotion scoring scale for sheep. The Veterinary Journal. 2009;180(2):189-194.

12. McLennan KM, Rebelo CJB, Corke MJ, et al. Development of a facia expression scale using footrot and mastitis as models of pain in sheep. Applied Animal Behaviour Science. 2016;176:19-26.

13. Muri K, Valle PS. Human-animal relationships in the Norwegian dairy goat industry: assessment of pain and provision of veterinary treatment (Part II). Animal Welfare. 2012;21(4):547-558.

14. Staffieri F, Driessen B, Lacitignola L, et al. A comparison of subarachnoid buprenorphine or xylazine as an adjunct to lidocaine for analgesia in goats; Veterinary Anaesthesia and Analgesia. 2009;36(5):502-511.

15. Lonardi C, Leach M, Gottardo F. The 'Grimace Scale': do piglets in pain change their facial expression? Proceedings of the Joint Meeting of the 5th European Symposium of Porcine Health Management and the 50th Anniversary Meeting of the Pig Veterinary Society of Great Britain, Edinburgh, UK. 2013.

16. Di Giminiani P, Brierley VLMH, Scollo A, et al. The Assessment of Facia Expressions in Piglets Undergoing Tail Docking and Castration: Toward the Development of the Piglet Grimace Scale. Front. Vet. Sci. 2016.

17. Göransson L. Porcine pain face - identifying visible characteristics of pain in pigs. 2016.

18. Valverde A, Doherty TJ. Chapter 105: Pain Management in Cattle and Small Ruminants. 2009. 